\title{
Kodamaea ohmeri
}

National Cancer Institute

\section{Source}

National Cancer Institute. Kodamaea ohmeri. NCI Thesaurus. Code C127725.

A species of yeast-like fungi in the phylum Ascomycota. K. ohmeri is a causative agent of fungemia in immunocompromised patients. This species is widely used in the food industry for fermentation of fruits, pickles and rinds. 